\title{
Políticas públicas de apoyo a la industria cultural de la historieta. Hacia un catálogo de casos
}

LUCIANO BROM

UNIVERSIDAD DE SAN ANDRÉS

ABSTRACT: This article work presents the first advances, research design and theoretical framework of my master's thesis on public policy supporting the production of graphic novels in Argentina and other countries relevant to the research. We have approached the production of graphic novels today as a branch of the publishing industry from the perspective of the theory of cultural industries, considering them as a kind of special commercial product that is both artistic, cultural and industrialized, which makes potential object of public policies by the State. The aim of this paper is to explore the active role of States in the development and maintenance of the cultural industry of graphic novels in Argentina and other countries, in order to compose a catalog of policy incentives for the graphic novel industry. Additionally, we seek to identify the various policies of material support from public to private sectors, that is, direct or indirect subsidies that helped to develop and/or sustain the local graphic novel industry in selected countries of America and Europe.

Keywords: cultural industry, public policy, graphic novel industry.

RESUMEN: El presente trabajo presenta los primeros avances y el diseño de investigación y marco teórico de mi tesis de maestría sobre políticas públicas de apoyo a la producción de historietas en Argentina y otros países relevantes para la investigación. Se planea analizar la producción de historietas en la actualidad como una rama de la industria editorial, desde la perspectiva de la teoría de las industrias culturales, considerando a la historieta como un tipo de producto especial que es a la vez un objeto artístico-cultural y un producto comercial industrializado, lo que la convierte en potencial objeto de políticas públicas por parte del Estado. El objetivo general del trabajo es explorar el rol activo de los Estados en el desarrollo y sostenimiento de la industria cultural de la historieta en Argentina y otros países, con el fin de componer un catálogo de políticas de incentivo a la industria de la historieta. Se busca caracterizar las diferentes políticas 
de apoyo material del sector público al privado, esto es, subsidios directos o indirectos que funcionaron o funcionan para el desarrollo y/o mantenimiento de la industria de la historieta local en ciertos países de América y Europa.

Palabras clave: industria cultural, políticas públicas, industria de la historieta.

La producción de historietas en Argentina tiene una larga historia de excelencia por su calidad y gran desarrollo comercial, en especial entre las décadas de 1940 y 1950 del siglo pasado (Vazquez, 2006 y 2010: 25). Sin embargo, un conjunto de factores desencadenaron una curva descendente desde fines de los cincuenta (Vazquez, 2010: 28), que llevaron a una larga crisis durante lo que restaba del siglo.

Es recién a partir del 2002 que se puede hablar de un resurgimiento de la producción de nuevos productos nacionales, de creciente calidad, que ha recibido el reconocimiento internacional tanto en premios como en traducciones y publicaciones en el exterior. En la actualidad, si bien han reaparecido publicaciones seriadas, ${ }^{1}$ como en el resto del mundo, la producción se concentra en el formato de publicaciones no periódicas, ajustándose a la definición del sistema ISBN para libros.

El propósito de la tesis de la que este trabajo es un avance, es analizar la producción de historietas en la actualidad, como una rama de la industria editorial argentina, desde la perspectiva de la teoría de las industrias culturales, considerando a la historieta como un tipo de producto especial que es a la vez un objeto artístico-cultural y un producto comercial industrializado, lo que la convierte en potencial objeto de políticas públicas. En este sentido, comparte muchas características con las industrias del libro y del cine en especial, entre otras industrias culturales que la bibliografía sobre el tema analiza.

En el caso de la industria de la historieta, a diferencia de lo sucedido con otras industrias culturales como las nombradas, el Estado argentino no ha tenido demasiada participación, ya sea por prejuicios ante un producto generalmente más industrial que artístico, ${ }^{2}$ o por ineficacia de los actores del mercado de la historieta para influir en el diseño de políticas públicas específicas para el sector.

1. Definidas e identificadas por el sistema ISSN (http://www.caicyt.gov.ar/issn/bfque-es).

2. «La historieta, en la encrucijada entre la técnica de reproducción masiva que supone su edición y el lenguaje artístico o artesanal que conforma su elaboración», reflexiona Vázquez (2010: 78). 
Esto provoca una falta de desarrollo en una industria cultural con una rica tradición en el país y un presente con recursos disponibles para lograr una producción de alta calidad, capaz de generar tanto recursos económicos concretos como «contenidos diversos y relatos y perspectivas propias sobre nuestras realidades sociales» (Puente, 2007: 51).

Existen países de gran desarrollo económico con políticas específicas de incentivos, así como países en desarrollo que, a menor escala, también dedican una porción de su presupuesto a la promoción de la producción de historieta nacional. Entre los casos del primer tipo se encuentran países como Italia, Francia, Canadá y España. En el segundo, Uruguay, Chile, Colombia, Ecuador y Brasil, entre otros.

Para ello, el proyecto propone estudiar el papel del Estado en el desarrollo y sostenimiento de la industria cultural de la historieta, en Argentina y en otras latitudes, con el fin de componer un catálogo de políticas de incentivo a la industria de la historieta. Frente a la multiplicidad de acciones estatales posibles para promocionar un objetivo definido, nos proponemos restringir la búsqueda a los casos de políticas activas de subsidio o créditos a la producción, sin perder de vista otras políticas públicas relevantes para el mercado de la historieta que hayan podido ser implementadas.

El producto del estudio será un banco/catálogo de políticas disponibles para la promoción de la industria nacional de la historieta, buscando, en última instancia, colaborar para dar respuesta al interrogante sobre qué políticas de este tipo serían viables en la realidad Argentina.

Pero la tarea de compilar un catálogo de políticas públicas de diferentes países cuyo objetivo sea promover la industria de la historieta nos plantea un número de interrogantes previo a la tarea de identificar y analizar las políticas en sí. En principio debemos definir qué entendemos por historieta, tanto en cuanto a medio gráfico de comunicación de masas, como a producto industrial definido por el formato de edición (libros, revistas, fascículos, etcétera).

Nuestra definición tendrá sin dudas ciertas similitudes y algunas diferencias con las caracterizaciones adoptadas por cada iniciativa concreta de promoción gubernamental. La definición de la historieta, y la categorización que se desprende de la misma, puede tener implicancias decisorias en el tipo de política implementada, e incluso en su diseño.

Así, tomar la historieta como una rama (industrializada) de las artes plásticas o como un medio inserto en la mecánica del mundo editorial plantea diferencias que abarcan toda la extensión de las políticas implementadas o proyectadas. A su vez, la discusión sobre una historieta nacional presenta ciertas dificultades toda vez que cualquier país en la actualidad se inserta en un escenario de mercado globalizado en que las viejas definiciones de lo nacional entran en conflicto. 
Por otro lado, una definición de políticas públicas se hace necesaria para circunscribir los casos a seleccionar para el análisis. No cualquier acción estatal puede entenderse como una política pública, y no todo accionar del Estado para promocionar la historieta es motivo de interés para nuestro estudio. El estudio de la génesis de esas políticas nos lleva a comprender los rasgos distintivos de las mismas, así como sus condiciones de oportunidad en un contexto de recursos por definición escasos, como es el de cualquier Estado nacional.

Las políticas públicas de promoción a la industria y la historieta, como parte de un conjunto de disciplinas artísticas de reproducción masiva, se encuentran bajo una misma mirada a partir de la popularización del concepto de industrias culturales. Un recorrido por el desarrollo del mismo será necesario para entender las condiciones de posibilidad del apoyo del Estado a un medio -no pocas vecesdespreciado como arte menor o simple entretenimiento.

Las políticas de promoción de las industrias culturales serán entonces objeto de análisis dentro del marco de las definiciones teóricas del estudio. Considerando los modos de producción de la historieta como producto mercantil, se hace necesario entender qué actores participan en la producción de las historietas, más allá de los creadores, condición necesaria pero no suficiente para el desarrollo del producto final, esto es, los ejemplares listos para la venta.

En cuanto al diseño de investigación, planeamos abrevar en la tradición del estudio de caso para cada política seleccionada, de manera de entender no solo comparativamente sino holísticamente cómo cada política fue planteada, llevada a cabo y cuáles fueron los resultados finales, ya sean estos positivos o negativos. Tomar cada caso como un todo nos permite entender cómo llegó el Estado en cuestión a interesarse por el apoyo a la industria, cuáles fueron los actores involucrados en las demandas y el diseño de la política implementada, cómo fue la implementación en sí, y los resultados obtenidos.

Más allá de las diferentes maneras en que se puede plasmar el apoyo a una industria cultural nacional (o a una industria nacional tradicional, en sentido más general), esto es, a las políticas de incentivos por medio de subsidios, créditos públicos con bajo o ningún interés, etcétera, nos interesa comenzar con algunas definiciones sobre las políticas públicas y su contexto de implementación según Evans (1996) y el ya clásico trabajo de Oscar Oszlak y Guillermo O’Donnell (1976). El Estado tiene una función central en el proceso de cambio estructural, afirma Evans (1996: 530). El reconocimiento de este papel central retrotrae, inevitablemente, a las cuestiones vinculadas con la capacidad del Estado (Evans, 1996: 530):

No se trata meramente de saber identificar las políticas correctas. La aplicación consistente de una política cualquiera, ya sea que apunte a dejar que los precios «alcancen su nivel correcto» o al establecimiento de una industria nacional, requiere 
la institucionalización duradera de un complejo conjunto de mecanismos políticos y [...] dicha institucionalización de ninguna manera puede darse por descontada.

La calidad institucional forma parte del contexto en que una política se diseña e implementa, influyendo directamente sobre el éxito de ambas etapas. Ese contexto es parte importante del análisis de una política pública según Oszlak y O’Donnell (1976), como veremos más adelante. Para estos autores existen dos enfoques sobre los estudios de políticas públicas. El enfoque más tradicional de las causas que originaron una política, que visualiza un Estado pasivo, dando respuesta a las demandas de «grupos sociales desde afuera», y la más novedosa, pero no por eso completamente adecuada, mirada sobre los impactos de las acciones estatales, que considera al Estado como autónomo e invisibiliza el contexto en el que las decisiones fueron tomadas (Oszlak y O’Donnell, 1976: 108).

Creemos posible focalizar el estudio de la dinámica de las transformaciones sociales siguiendo la trayectoria de una cuestión a partir de su surgimiento, desarrollo y eventual resolución. Las sucesivas políticas o tomas de posición de diferentes actores frente a la cuestión y la trama de interacciones que se van produciendo alrededor de la misma definen y encuadran un proceso social.

Solo algunas de las demandas y necesidades de una sociedad son problematizadas como «cuestiones» por ciertas clases, fracciones, grupos o individuos estratégicamente situados y son incorporadas a la agenda de problemas socialmente vigentes. Sin embargo, no todas las cuestiones reciben atención del Estado, ya que algunas son libradas a la sociedad civil, cuando ni el Estado ni los actores afectados estiman necesaria u oportuna la intervención estatal. En el caso de la promoción de la historieta como una industria cultural, esta es una variante históricamente repetida en nuestro país, en el que el Estado ha sido un actor ausente en el desarrollo, apogeo y declive de la industria editorial de la historieta (Oszlak y O’Donnell, 1976: 111):

Deberíamos encarar nuestros estudios analizando el periodo previo al surgimiento de la cuestión. Nos interesa aprender quién la reconoció como problemática, cómo se difundió esta visión, quién y sobre la base de qué recursos y estrategias logró convertirla en cuestión. El examen de este «periodo de iniciación» puede enriquecer nuestro conocimiento sobre el poder relativo de diversos actores, sus percepciones e ideología, la naturaleza de sus recursos, su capacidad de movilización, sus alianzas y conflictos y sus estrategias de acción política. Nos sirve no solo para interpretar eventos posteriores, sino también para iluminar algunos de los problemas más generales sobre las características del Estado, y las nuevas modalidades que asumen sus patrones de interacción con la sociedad civil. 
Siendo este un proyecto de tesis de una maestría sobre administración pública y políticas públicas, este último punto señalado por los autores no es accesorio, sino parte del interés general que tiene analizar políticas específicas, como, en este caso, las de apoyo a la industria de la historieta:

Una política estatal es una toma de posición del Estado frente a una cuestión. Por lo general incluye decisiones de una o más organizaciones estatales, simultáneas o sucesivas a lo largo del tiempo, que constituyen el modo de intervención del Estado frente a la cuestión. De aquí que la toma de decisión no tiene por qué ser unívoca, homogénea ni permanente [...].

El análisis de las variaciones de la acción estatal a lo largo del tiempo se convierte así en punto clave del método de investigación. No hablamos de una fotografía de las políticas implementadas, sino de un proceso de intervención estatal, ya sea en marcha o cerrado, con resultados positivos, negativos o neutros (Oszlak y O’Donnell, 1976: 112-114):

Concebimos una política estatal (o pública) como un conjunto de acciones y omisiones que manifiestan una determinada modalidad de intervención del Estado en relación con una cuestión que concita la atención, interés o movilización de otros actores en la sociedad civil [...] La política estatal no constituye ni un acto reflejo ni una respuesta aislada, sino más bien un conjunto de iniciativas y respuestas manifiestas o implícitas, que observadas en un momento histórico y en un contexto determinados permite inferir la posición - predominante- del Estado frente a una cuestión que atañe a sectores significativos de la sociedad [...] El Estado aparece como un actor más en el proceso social desarrollado en torno a una cuestión. Su intervención supone 'tomar partido', sea por acción u omisión. Una toma de posición activa puede implicar desde iniciar la cuestión y legitimarla, a acelerar algunas de sus tendencias, moderar otras o simplemente bloquearla. En los casos de inacción, el Estado puede haber decidido esperar a que la cuestión y la posición de los demás actores estén más nítidamente definidas, dejar que se resuelva en la arena privada entre las partes involucradas o considerar que la inacción constituye el modo más eficaz de preservar o aumentar los recursos políticos del régimen. Puede así imaginarse una multiplicidad de situaciones en las que el Estado - a través de diversos aparatos o instancias- decide insertarse (o no) en un proceso social, en una etapa temprana o tardía de su desarrollo, con el objeto de influir sobre su curso, asumiendo posiciones que potencialmente pueden alterar la relación de fuerzas de los actores involucrados en torno a la cuestión, incluyendo al propio Estado.

El resto de los actores involucrados objetiva o subjetivamente en una cuestión pueden (o no) movilizarse activamente en la defensa o cuestionamiento de la situación. Esto dependerá de su percepción de la situación, de que lo consideren natural, o de sus posibilidades de movilización por acceso a los recursos o miedo 
a ser reprimidos, en casos más extremos. Las tomas de posición de otros actores, además del Estado, pueden influir considerablemente en el proceso de resolución de las cuestiones.

Por ello se sugiere estudiar procesos sociales analizando las prácticas de diferentes actores, aglutinadas en torno a cuestiones que definen la naturaleza, intensidad y límites de un área de acción (y, habitualmente, de conflicto) social. Sin embargo, se advierte que el conjunto de políticas privadas y estatales se entrelazan en un complejo proceso social que hace difícil establecer con precisión qué proporción del cambio social observado puede ser atribuido a cada una.

Los autores identifican dos grandes niveles de contexto de una política estatal. El primero consiste en «aquel conjunto de factores extrínsecos al objeto más específico de investigación (las políticas estatales) que es indispensable para la comprensión, descripción y explicación de aquel objeto y sus efectos sobre otras variables». Estos factores incluyen las cuestiones que se intenta resolver, las condiciones de surgimiento de la cuestión, y las políticas adoptadas por actores sociales privados (Oszlak y O’Donnell, 1976: 125). Pero este primer contexto es insuficiente sin considerar la agenda de cuestiones, un segundo nivel de contexto.

¿Qué problemas merecen ser cuestiones? ¿Quiénes y cómo definen la agenda (el espacio problemático de una sociedad) de las mismas? El problema analítico es el conflicto y las coaliciones que se generan alrededor de las cuestiones que deben integrar la agenda, como consecuencia de las limitaciones para prestar atención a todas las cuestiones suscitables y a la diferencia de intereses, concepciones y recursos de los actores para imponer social y políticamente sus agendas.

La racionalidad acotada de los actores (debido a su incapacidad de procesar toda la información políticamente relevante de la agenda, al desconocimiento de las conexiones causales entre cuestiones, o la imposibilidad de predecir el comportamiento del resto de los actores) no impide, sin embargo, deducir o entender las decisiones en función de posiciones pasadas o presentes (con quién está aliado y en conflicto en otras cuestiones, qué recursos tiene invertidos en otras cuestiones, cuál es la movilidad de los mismos para atender otras cuestiones, etcétera).

El conocimiento de este segundo nivel de contexto suele ser necesario para explicar las políticas estatales que estudiamos específicamente, afirman los autores Oszlak y O'Donnell (1976: 128). Se plantean entonces cuatro niveles en los que organizar la investigación: I) las políticas estatales mismas, II) la cuestión a las que ellas se refieren, generando un proceso social que contiene a las políticas estatales y las privadas referidas a la cuestión, III) la agenda de cuestiones y IV) la estructura social, como contexto global más estático. Los dos primeros niveles serán, comúnmente, objeto de la investigación (Oszlak y O’Donnell, 1976: 130). 
Los puntos I y II son lo que Oszlak y O’Donnell consideran el tema propio de la investigación y el ámbito empírico en el que nos corresponderá recoger información.

Pero, ¿de qué políticas públicas hablamos cuando nos referimos a la historieta? ¿Y cuáles de ellas serán el corpus de la investigación planteada? En Francia, por ejemplo, Thierry Gorensteen (2006: 133) nombra varias vertientes de políticas estatales ensayadas en apoyo a la Bande Dessinée. Primero tomada por la Dirección de Artes Plásticas, la gestión de la ayuda a la historieta francesa fue luego derivada a la Dirección del Libro y la Lectura, por medio del Centro Nacional del Libro (CNL). El CNL subvenciona con premios a los creadores e investigadores, ayuda a los editores de libros y revistas, da créditos para la conformación y desarrollo de fondos de historietas en bibliotecas, y da apoyo, finalmente, al Festival de Angulema entre otros.

Relevar todo tipo de apoyo estatal a la historieta excedería largamente los recursos disponibles para la investigación, por lo que limitamos el estudio a las políticas que implican directamente una ayuda a los editores. Esto es, el apoyo a la producción de historietas por parte del Estado, directamente a los actores encargados de la tarea empresarial. Tarea imprescindible, por otra parte, para la producción del producto final de la industria cultural ya mencionada: el libro de historietas.

Para acotar el campo de estudio, debemos explicitar la definición que usaremos de industria cultural, entre las múltiples variantes y debates al respecto. Según Getino (2003: 21), el concepto de industrias culturales alude al:

[...] conjunto de actividades relacionadas directamente con la creación, la fabricación, la comercialización y los servicios de productos o bienes culturales, en el ámbito de un país o a nivel internacional. Los rasgos distintivos de estas industrias son semejantes a los de cualquier otra actividad industrial y se basan en la serialización, estandarización, la división del trabajo y el consumo de masas. A diferencia de otras, no se trata de productos para el uso o el consumo físico, sino de bienes simbólicos (obras literarias, musicales, cinematográficas, plásticas, periodísticas, televisivas, etc.) que para acceder a la percepción (consumo) de los grandes públicos, debe procesarse o manufacturarse industrialmente para adoptar la forma de un libro, un disco, una película, una publicación periódica, una reproducción o un programa de televisión.

Para Getino (2008: 81), los agentes principales de la industria del libro son: los autores, que elaboran las obras que los editores seleccionan; las editoriales, empresas dedicadas a seleccionar y publicar las obras que formarán parte de su catálogo, ocupándose de la producción y distribución en un período de tiempo y territorio determinados, agrupadas, en Argentina, principalmente en la Cámara Argentina del Libro (CAL), la cual administra también el siste- 
ma ISBN argentino; las empresas distribuidoras, que reciben generalmente en consignación los libros que le suministra el editor -quién también puede ser distribuidor de su propia producción- y se encargan de colocarlos en los canales de venta; y los canales de venta, por último, que tienen la función de hacer que el libro llegue a los lectores, convirtiéndose en los espacios de comercialización directa con el público.

A estos actores, Getino suma las industrias auxiliares, como empresas gráficas, quienes se ocupan del proceso industrial de fabricación del libro (fotocomposición, impresión y encuadernación) a partir de los originales y diseño que les proporcionan las empresas editoriales. Para ello, se ocupan de adquirir en el mercado los insumos necesarios (papel, vegetales, etc.).

También intervienen diferentes empresas de servicios de corrección, diseño gráfico, promoción y publicidad. E indirectamente, las empresas fabricantes (o importadoras) de papel, las empresas fabricantes o importadoras de tecnología e insumos para la producción de libros, y el Estado, quien regula las actividades del sector en materia de derechos de autor, y fomenta la producción y divulgación de libros en el sector educativo y en bibliotecas públicas y populares. Dentro del sector público, el INDEC, a su vez, procesa parte de la información del sector referida a importaciones y exportaciones de libros, papel, máquinas e insumos, y a la facturación de las empresas.

Como Getino se refiere específicamente a la industria editorial en general, no atiende a las particularidades de la producción de libros de historieta, a saber (y especialmente en el caso de la Argentina): los autores en algunos casos comprenden dos o más personas, entre guionistas y dibujantes (e incluso coloristas, y otros sub-rubros del dibujo), aunque en la actualidad existen muchos artistas integrales, esto es, una sola persona que se hace cargo de la totalidad del acto creativo. Con respecto a los editores, si bien existen empresas consolidadas, la gran mayoría de la producción proviene de empresas pequeñas o unipersonales, por lo que es común referirse a los editores, en lugar de hablar de editoriales.

Los editores pueden, en algunos casos, encargar trabajos a los artistas, y no solo seleccionar el material ya creado. En la CAL, por su parte, estan registradas algunas editoriales de historieta, pero no todas, sobre todo porque estas son micro o pequeñas empresas, y la participación en la CAL es arancelada. Los canales de venta de la historieta se diferencian en tres: puestos de diarios y revistas, el tradicional punto de venta de la revista de historieta, pero que en los últimos años ha extendido su oferta a libros de todo tipo, incluidos los de historietas; librerías tradicionales, con distintos grados de apertura hacia los productos de la historieta; y las comiquerías, locales especializados surgidos en la década del noventa con la convertibilidad que permitía la importación de material extranjero y que aun 
sobreviven, aunque diversificando su oferta de productos, en la que la historieta (y sobre todo la historieta nacional) representa una pequeña parte de las ventas.

Estas últimas se concentran en la ciudad de Buenos Aires, pero existen locales también en las principales ciudades de cada provincia. Al existir tal segmentación de canales de venta, las distribuidoras exclusivas de historietas se encuentran bien diferenciadas según el canal que abastecen: La Revistería, distribuidora, importadora y con sello propio, que distribuye a librerías y comiquerías; Districomix, una empresa unipersonal que se encarga del vínculo exclusivamente con comiquerías; y las distribuidoras para kioscos de diarios y revistas de la ciudad de Buenos Aires y del interior del país. ${ }^{3}$

Existen otros actores que si bien no forman parte del circuito de producción y comercialización directamente, pueden influir de manera indirecta en diferentes puntos de la cadena de comercialización, como periodistas (sobre todo los que tienen espacio en medios de comunicación de gran alcance como diarios de tirada nacional), galerías de arte que incluyen obras de historietistas como parte de sus exposiciones, escuelas de dibujo e historieta, asociaciones no gubernamentales organizadoras de festivales y eventos de promoción de la historieta, ${ }^{4} \mathrm{O}$ investigadores académicos trabajando en la materia.

Como afirma Eugene Bardach (1998: 102), «en una investigación de políticas, casi todas las fuentes de información, datos e ideas provienen generalmente de dos tipos de fuentes: documentos y personas». Para relevar cada política de promoción de la industria cultural de la historieta debemos identificarla, a partir de informantes clave y documentos de promoción institucional de las organizaciones responsables de los programas, estudiar su contenido por medio del análisis de los documentos que rigen la política en sí (leyes, reglamentos, decretos, circulares, etc.) o el relato del caso por parte de informantes clave en caso de ser políticas de poca institucionalización formal, y explorar el contexto para el que fue diseñada e implementada cada política, por medio de informantes clave, notas periodísticas y/o eventuales documentos de organizaciones de la industria cultural de la historieta en los que se discuta el rol del Estado en la misma.

Los informantes clave en cada país serán editores de historieta, autores con una mirada reflexiva sobre el aspecto comercial de su trabajo, periodistas y críticos especializados, investigadores y policy-makers. El énfasis en los informantes como núcleo central de la investigación se basa en el hecho de que las políticas orientadas a un sector menor dentro del esquema productivo de un país en ge-

3. Existen algunas distribuidoras de libros que están expandiendo su alcance a libros de historieta, como Nuestra América, pero son aun experiencias incipientes.

4. Como el caso de la Asociación Civil Viñetas Sueltas, organizadora del Festival Internacional de Historietas en Buenos Aires, o Banda Dibujada, de promoción de la historieta infantil y juvenil. 
neral no son regidas por leyes o decretos de alto nivel, sino que surgen como iniciativas de un área específica, y en muchos casos tienen poco basamento documental, por lo que explotar el conocimiento de los funcionarios responsables o de los beneficiados por las políticas de incentivo daría mayor riqueza al estudio.

El objetivo es obtener información de las políticas identificadas, de manera de lograr un insumo que consiste en una matriz de datos de los casos, en el que se sistematiza la información para el posterior análisis detallado. Para ello, se cuenta con fuentes documentales y entrevistas presenciales o virtuales con actores relevantes de cada mercado estudiado.

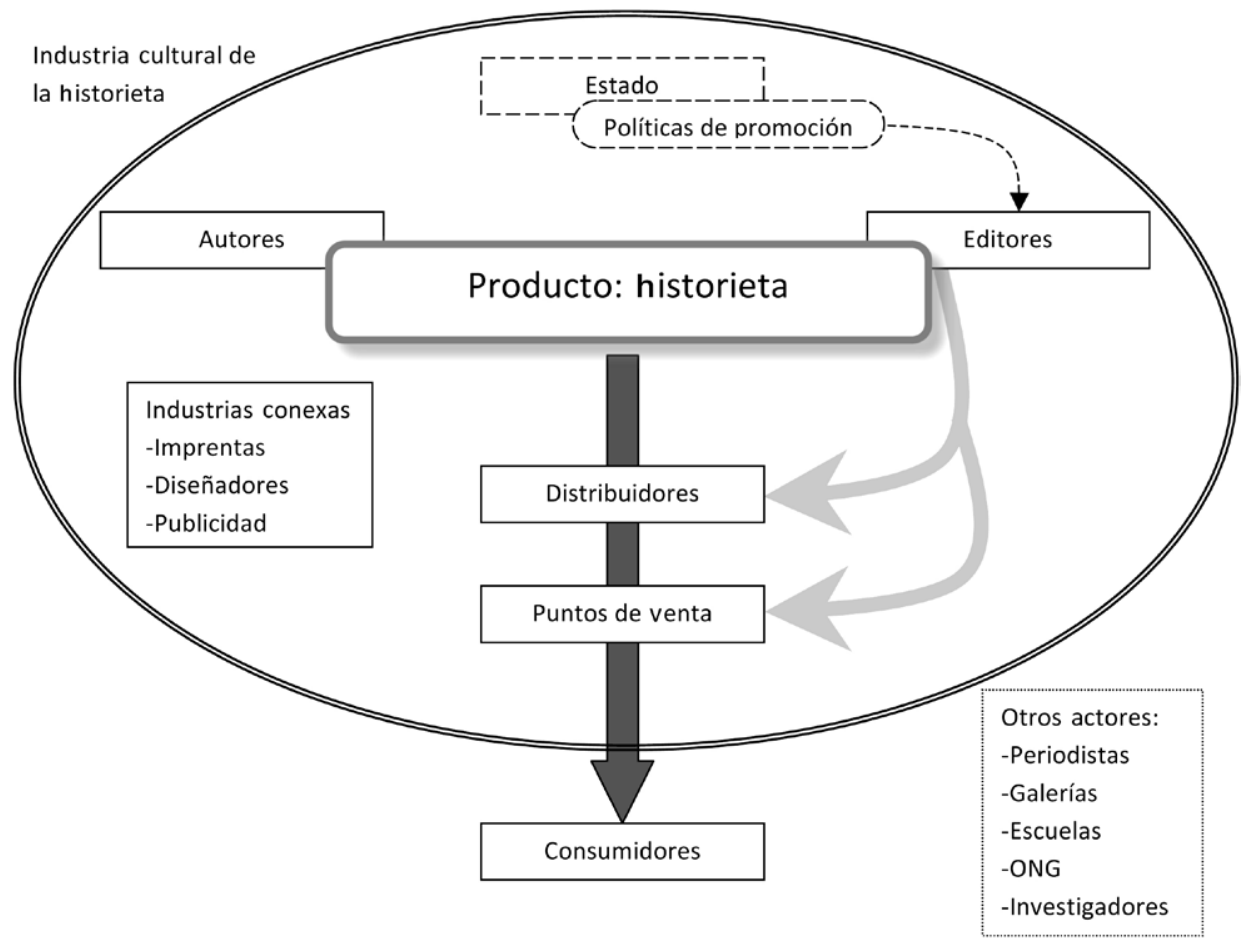

\section{Esquema conceptual}




\section{Referencias bibliográficas}

BARDACH, E. (1998): Los ocho pasos para el análisis de politicas públicas. Un manual para la práctica, México DF, Cide-Porrúa.

Evans, P. (1996): «El estado como problema y como solución», Revista Desarrollo Económico, 35, n. ${ }^{\circ}$ 140: 529-562.

- (2003): Industrias culturales: mercado y políticas públicas en Argentina, Buenos Aires, Secretaría de Cultura/ciccus.

Getino, O. (2008): El capital de la cultura: las industrias culturales en la Argentina, Buenos Aires, CICCUS.

OszlaK, O.; G. O’Donnell (1976): Estado y politicas estatales en América Latina: hacia una estrategia de investigación, Buenos Aires, Cedes.

Puente, S. M. (2007): Industrias culturales, Buenos Aires, Prometeo Libros.

Groensteen, T. (2006): Un objet culturel non identifié, Paris, Editions de l'an 2.

VAzQuez, L. (2006): «Tiempo varado. Historieta, Arte y Cultura en la Argentina del siglo XX». Recuperado el 20 septiembre de 2009 de:

www.tebeosfera.com/documentos/documentos/tiempo_varado_historieta_ arte_y_cultura_en_la_argentina_del_siglo_xx.html

- (2010): El oficio de las viñetas. La industria de la historieta argentina, Buenos Aires, Paidós. 